\title{
The role of the gut microbiota in the dietary niche expansion of fishing bats
}

\author{
Ostaizka Aizpurua ${ }^{1 *} \mathbb{D}$, Lasse Nyholm¹, Evie Morris², Gloriana Chaverri ${ }^{3,4}$, L. Gerardo Herrera Montalvo ${ }^{5}$, \\ José Juan Flores-Martinez ${ }^{6}$, Aiqing Lin' ${ }^{7}$, Orly Razgour ${ }^{2}$, M. Thomas P. Gilbert ${ }^{1,8}$ and Antton Alberdi ${ }^{1}$
}

\begin{abstract}
Background: Due to its central role in animal nutrition, the gut microbiota is likely a relevant factor shaping dietary niche shifts. We analysed both the impact and contribution of the gut microbiota to the dietary niche expansion of the only four bat species that have incorporated fish into their primarily arthropodophage diet.

Results: We first compared the taxonomic and functional features of the gut microbiota of the four piscivorous bats to that of 11 strictly arthropodophagous species using 165 rRNA targeted amplicon sequencing. Second, we increased the resolution of our analyses for one of the piscivorous bat species, namely Myotis capaccinii, and analysed multiple populations combining targeted approaches with shotgun sequencing. To better understand the origin of gut microorganisms, we also analysed the gut microbiota of their fish prey (Gambusia holbrooki). Our analyses showed that piscivorous bats carry a characteristic gut microbiota that differs from that of their strict arthropodophagous counterparts, in which the most relevant bacteria have been directly acquired from their fish prey. This characteristic microbiota exhibits enrichment of genes involved in vitamin biosynthesis, as well as complex carbohydrate and lipid metabolism, likely providing their hosts with an enhanced capacity to metabolise the glycosphingolipids and longchain fatty acids that are particularly abundant in fish.
\end{abstract}

Conclusions: Our results depict the gut microbiota as a relevant element in facilitating the dietary transition from arthropodophagy to piscivory.

Keywords: Chiroptera, Diet, Microbiome, Microorganism, Niche shift, Piscivorous, Trophic niche

\section{Background}

Given their fundamental roles in acquiring the energy needed for animals to develop, survive and reproduce, traits associated with diet are expected to be under strong selection pressure [1]. Thus, diversification of dietary niches is one of the prevailing processes in animal evolution [2]. However, when a novel dietary resource differs considerably from the original, the resulting trophic change might need to be accompanied with a physiological adaptation, so that animals can make the most

*Correspondence: ostaizka.aizpurua@sund.ku.dk

${ }^{1}$ Center for Evolutionary Hologenomics, GLOBE Institute, University of Copenhagen, 1353 Copenhagen, Denmark

Full list of author information is available at the end of the article of the nutritional value of the novel food [3]. The gut microbiota has been identified as a key element for such processes $[4,5]$, first, because microorganisms can complement the digestive capabilities of the host by extracting and metabolising dietary ingredients that the hosts' enzymatic toolbox are unable to process; and second, because microorganisms provide essential compounds like vitamins and short-chain fatty acids to their hosts $[6,7]$. Many microorganisms that reside in the intestinal tract of animals are acquired directly through the diet [8], and some contribute to the metabolism of dietary ingredients, thus providing the host with the capacity to better exploit the nutritional potential of the food [9].

Bats are an excellent system with which to study the role of microorganisms in such dietary shifts, due to original author(s) and the source, provide a link to the Creative Commons licence, and indicate if changes were made. The images or other third party material in this article are included in the article's Creative Commons licence, unless indicated otherwise in a credit line to the material. If material is not included in the article's Creative Commons licence and your intended use is not permitted by statutory regulation or exceeds the permitted use, you will need to obtain permission directly from the copyright holder. To view a copy of this licence, visit http://creativecommons.org/licenses/by/4.0/. 
recurrent invasion into non-arthropod feeding niches [10-12], and their digestive capabilities. Despite having reduced the length of their intestinal tract as a weightsaving adaptation to flight, the digestion and absorption capacities of bats are comparable to those of similar-sized non-flying mammals [13]. While some bat species have specialised in consuming a single food resource (e.g. blood-feeding bats, [14]), others have incorporated new feeding resources into their ancestral arthropod-based diet [10], thus expanding their dietary niche. One example of the latter is the case of the four well-known piscivorous (also known as fishing) bat species [15], which have independently developed fishing behaviour in four geographical areas across the planet (Fig. 1). However, the four species exhibit different patterns of fish consumption, ranging from widespread (occurring in most of the individuals) and common (up to 90\%) in Noctilio lepori$n u s$, to restriction to only certain colonies and seasons in the three species of Myotis piscivorous bats; namely, M. pilosus, M. vivesi and M. capaccinii [15]. Despite this fascinating twist to their life history, the spatio-temporal patterns, causes and consequences of fishing behaviour largely remain unexplored.

Given there are considerable nutritional differences between arthropods and fish [16], we predicted that piscivorous bats host a gut microbiota that is distinct from that of their strictly arthropodophagous (i.e. organisms that prey on arthropods, [17]) counterparts. Our rationale is based on the hypothesis that this microbiota could help confer the digestive capability required to acquire nutrients from fish flesh. We also explore whether the new repertoire of microbial functions has been acquired from microorganisms associated with the new food resource. Finally, as piscivory is often limited to certain colonies, and perhaps certain individuals, rather than established among all individuals within a species [15], we also analysed whether microbiota traits associated with piscivory are restricted to actively fishing individuals, or established across populations of piscivorous bats. Overall, we aim to understand not only how a dietary shift affects gut microbial communities, but also how microbiota changes can facilitate such a trophic niche expansion.

\section{Results and discussion}

We first used targeted amplicon sequencing of the bacterial 16S rRNA gene to generate and analyse the taxonomic and functional gut microbiota profiles of 47 individuals belonging to 11 strict arthropophagous bat species and 37 individuals belonging to well-known piscivorous colonies of the four facultative piscivorous species (e.g. consuming both arthropods and fish, hereafter referred to simply as piscivorous for the sake of clarity) (Fig. 1, see Additional file 1: Table S1.1). In a second step, we increased the resolution of our analyses in the piscivorous bat Myotis capaccinii, by adding more individuals from three allegedly non-piscivorous colonies to the analysis, as well as incorporating shotgun sequencing data for direct fish DNA quantification and inference of functional microbiota features. To gain further insights into the origin of gut microorganisms, we also analysed the gut microbiota of $M$. capaccinii's fish prey (Gambusia holbrooki). We then implemented an integrative approach that included Hill numbers-based diversity analyses [18], multivariate statistics, ensemble machine learning modelling [19] and enrichment analyses, in

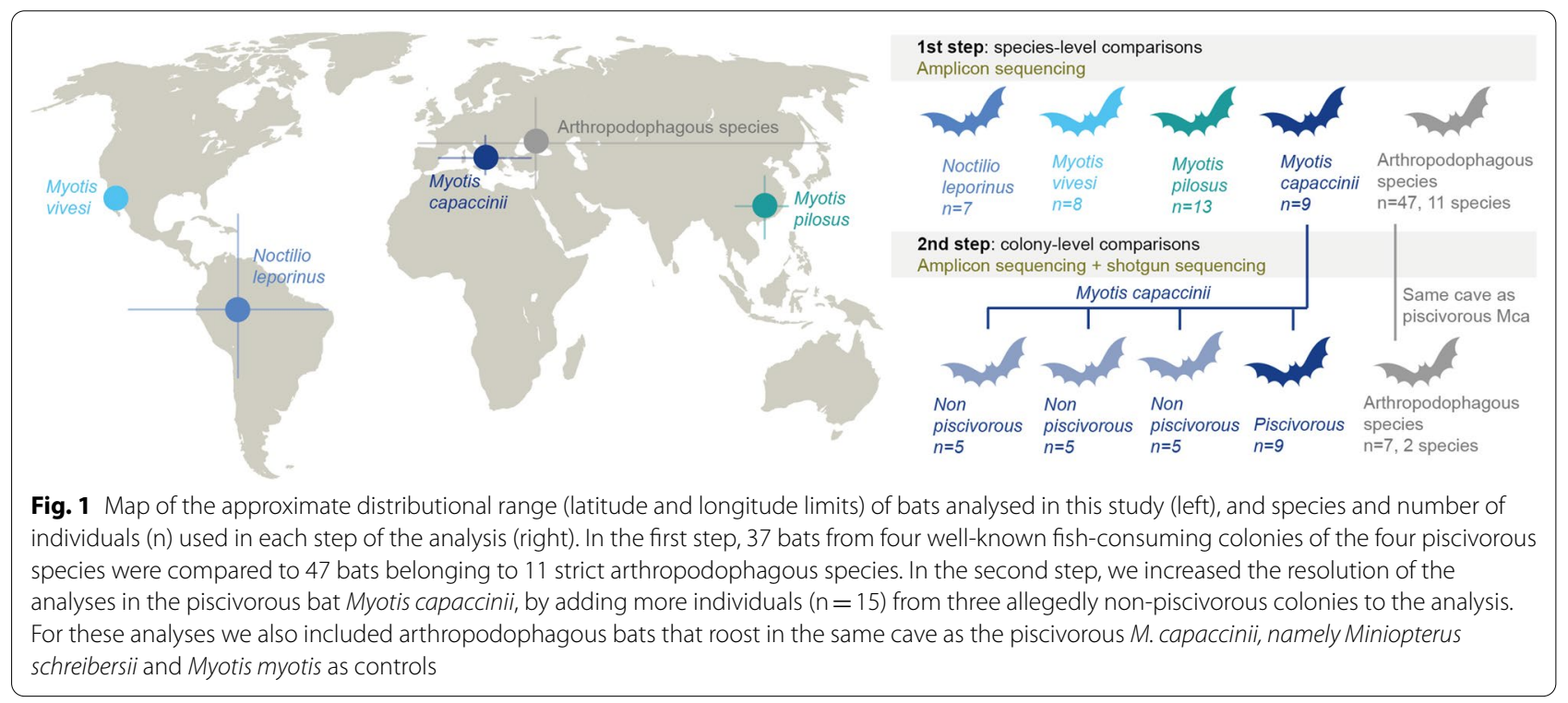


order to (1) identify the gut microbiota patterns behind this trophic shift, (2) unveil the factors shaping gut microbial communities, and (3) understand the implications of hosting different microbiotas for bats.

\section{Piscivorous bats host unique gut microbiotas}

Taxonomic characterisation of the gut microbiota associated with the 15 bat species studied using amplicon sequencing exhibited an overall microbial community that was comprised of 27 phyla, which was principally dominated by Proteobacteria $(56.36 \pm 33.41 \%$; mean \pm standard deviation $)$ and Firmicutes $(22.16 \pm 28.78 \%)$ (see Additional file 1: Fig. S1.1). These overall patterns are in accordance with previous observations [20-23]. We observed large interindividual (overall amplicon sequence variant (ASV) turnover rate across individuals within species of $87.98 \pm 0.08 \%$ ) and interspecific variability $\left(\right.$ PERMANOVA $_{\mathrm{U} 12}: \mathrm{R}^{2}=0.195$, $p \quad$ value $=0.001 ; \quad$ PERMANOVA $_{\mathrm{U} 12}: \quad \mathrm{R}^{2}=0.198, \quad p$ value $=0.001$, see Additional file 1: Table S1.4) in their gut microbiota, indicating the community likely responds to the wide breadth of ecological and evolutionary features embedded within the animals studied. However, this variability did not mask diet-related patterns. In particular, despite exhibiting similar microbial diversity values (Wilcoxon: $p$ value $>0.05$ at different $\mathrm{q}$ values, see Additional file 1: Table S1.2 and Fig. S1.2), both the analysis of variance $\left(\right.$ PERMANOVA $_{\mathrm{U} 12} \mathrm{R}^{2}=0.057, p$ value $=0.001$; PERMANOVA $_{\tilde{U} 12}: \mathrm{R}^{2}=0.072, p$ value $=0.001$, see Additional file 1: Table S1.4 for PERMANOVA analysis on other neutral and phylogenetic Hill number of order of diversity 0 ) and the ensemble machine learning classification (accuracy $=1$ ) showed that piscivorous bats have compositionally characteristic gut microbial communities that differ from that of arthropodophagous bats. The distinctiveness of piscivorous bats is mainly attributed to five bacteria genera, namely Aeromonas, Plesiomonas, Photobacterium, Cetobacterium and Paraclostridium, as these were significantly enriched in piscivorous bats (Fig. 2), and were also the bacteria that contributed the most to the ensemble predictive models of piscivorous bats (see Additional file 1: Table S1.3).

\section{The gut microbiota of piscivorous bats shows limited signature of convergence}

We did not detect a core microbiota (i.e. bacteria represented in $>90 \%$ of individuals) at ASV- or genus-level, among piscivorous or among arthropodophagous bats. Accordingly, the relative representation of bacterial taxa characteristic of piscivorous bats differed markedly across species, ranging from marginal presence in some, to being the dominant taxon in others. Consequently, while the resulting gut microbiotas of piscivorous bats were different from those of arthropodophagous species, they did not converge into one characteristic type of microbial community associated with piscivory (PERMANOVA $_{\mathrm{U} 12}: \mathrm{R}^{2}=0.312, p$ value $=0.001$, Fig. 3 , see Additional file 1: Table S1.4). Similar patterns have been described as convergence in other groups of phylogenetically distant species with similar diets, such as ant-eating mammals [24]. However, we argue that convergence entails not only exhibiting distinctive microbial communities, but also an increase in their similarity, something that is not observed in any of the cases. Extending our work by generating deep shotgun metagenomic data from the different fishing bat species would enable ascertaining whether the observed microbiota variations entail functional convergence $[25,26]$.

The two piscivorous species with the highest microbial community resemblance are $M$. capaccinii and $M$. pilosus

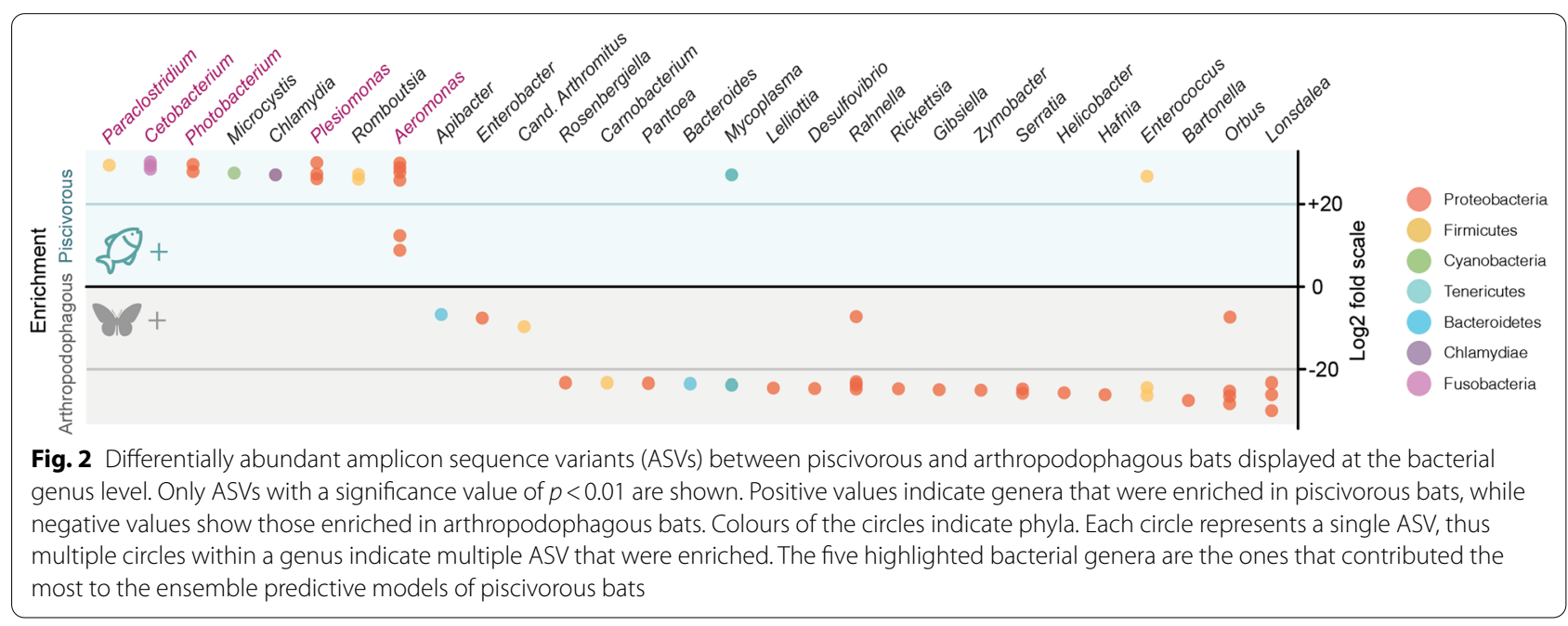




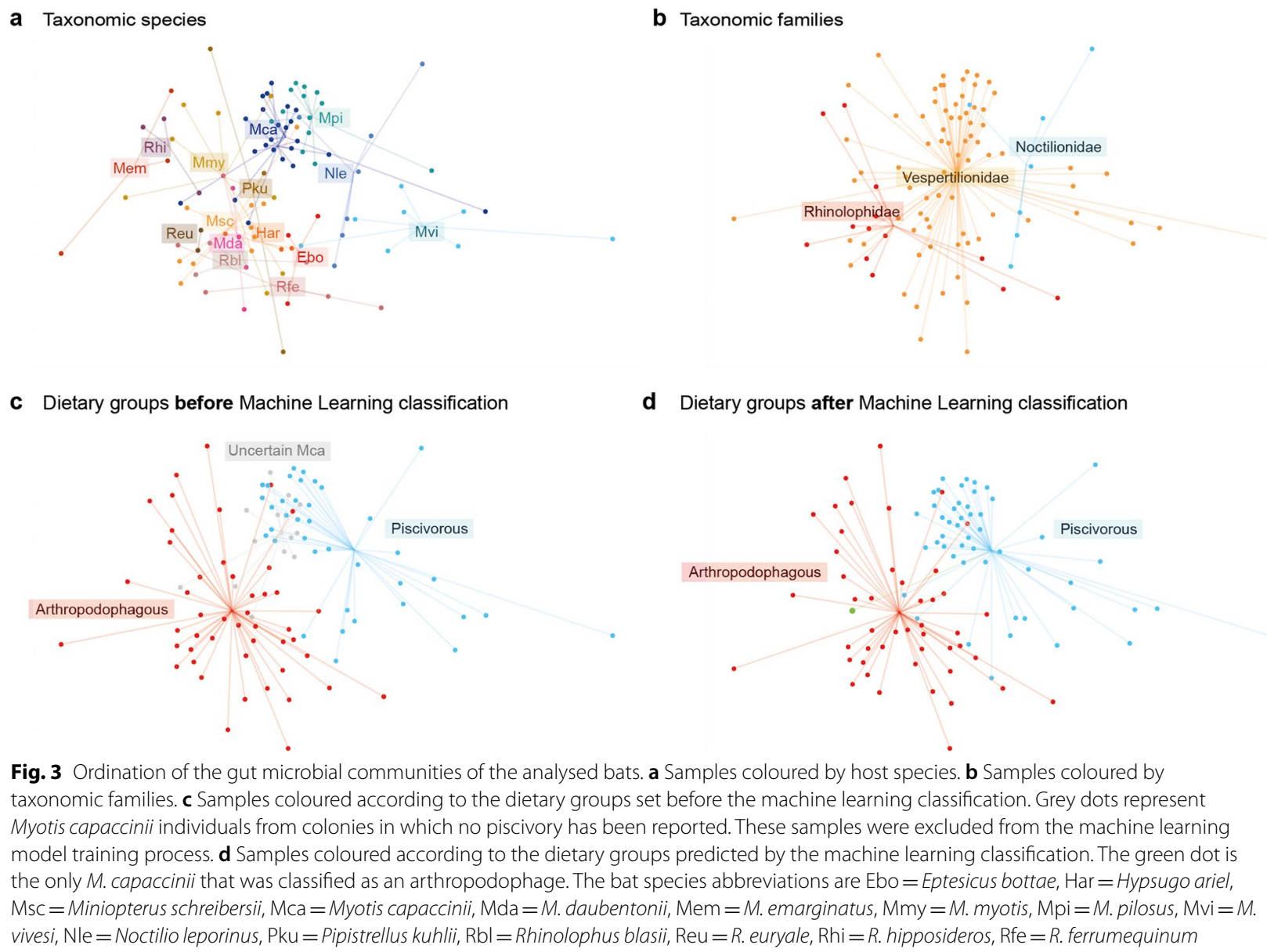

a Taxonomic species

C Dietary groups before Machine Learning classification

b Taxonomic families

d Dietary groups after Machine Learning classification

Fig. 3 Ordination of the gut microbial communities of the analysed bats. a Samples coloured by host species. b Samples coloured by taxonomic families. c Samples coloured according to the dietary groups set before the machine learning classification. Grey dots represent Myotis capaccinii individuals from colonies in which no piscivory has been reported. These samples were excluded from the machine learning model training process. $\mathbf{d}$ Samples coloured according to the dietary groups predicted by the machine learning classification. The green dot is the only M. capaccinii that was classified as an arthropodophage. The bat species abbreviations are Ebo=Eptesicus bottae, Har = Hypsugo ariel, $\mathrm{Msc}=$ Miniopterus schreibersii, $\mathrm{Mca}=$ Myotis capaccinii, $\mathrm{Mda}=\mathrm{M}$. daubentonii, Mem =M. emarginatus, $\mathrm{Mmy}=\mathrm{M}$. myotis, Mpi=M. pilosus, Mvi=M. vivesi, $\mathrm{Nle}=$ Noctilio leporinus, Pku $=$ Pipistrellus kuhlii, $\mathrm{Rbl}=$ Rhinolophus blasii, Reu $=$ R. euryale, $\mathrm{Rhi}=R$. hipposideros, Rfe $=$ R. ferrumequinum

(Fig. 3a, see Additional file 1: Fig. S1.3), due to the high representation of Aeromonas and Cetobacterium in both species (Fig. 4). M. capaccinii and M. pilosus are the two piscivorous bats with both the highest phylogenetic [27] and ecological resemblance, as they exclusively forage in freshwater habitats $[28,29]$. However, the reason for the dominant bacteria to be different in each bat -Aeromonas (Proteobacteria) in M. capaccinii and Cetobacterium (Fusobacteria) in M. pilosus - might be that the bats inhabit different habitats (M. capaccinii is found in Mediterranean and M. pilosus in temperate-subtropical habitats), and consume different species of fish [29, 30].

The other two piscivorous bat species exhibited rather different microbial communities, probably shaped by different extrinsic and intrinsic forces. The microbiota of $M$. vivesi stands out for an overall dominance of Firmicutes (49.07 $\pm 42.15 \%$, see Additional file 1: Fig. S1.1), a high representation of Photobacterium (Proteobacteria), and an absence of Aeromonas and Cetobacterium (Fig. 4). We believe this relates to the fact that $M$. vivesi is the sole species to specialise in foraging in the ocean [31]. The high salinity of the water and type of prey might modify the physicochemical conditions of the intestinal environment, up to the point of shaping a completely different microbiota [32,33]. This is supported by observations of higher prevalence of Firmicutes compared to Proteobacteria in salinity gradients [34]. Increased incidence of Firmicutes has also been related to the consumption of the Engraulidae fish [35] that $M$. vivesi consumes [36], due to their high content of polyunsaturated fatty acids that are known to promote intestinal enrichment of Firmicutes [37]. Furthermore, the pool of microbial species is different in salt and fresh water [38], for instance Photobacterium are ubiquitous in the oceans [39] and often found in symbiotic relationships with fish within the dietary spectrum of $M$. vivesi $[40,41]$.

The gut microbiota of N. leporinus is also different from that of other piscivorous species, probably due to a combination of the frequency of piscivory, the fish species consumed, the foraging environment, and its evolutionary distinctiveness. The incidence of fish in the diet of $N$. leporinus is considerably larger than in the three Myotis 


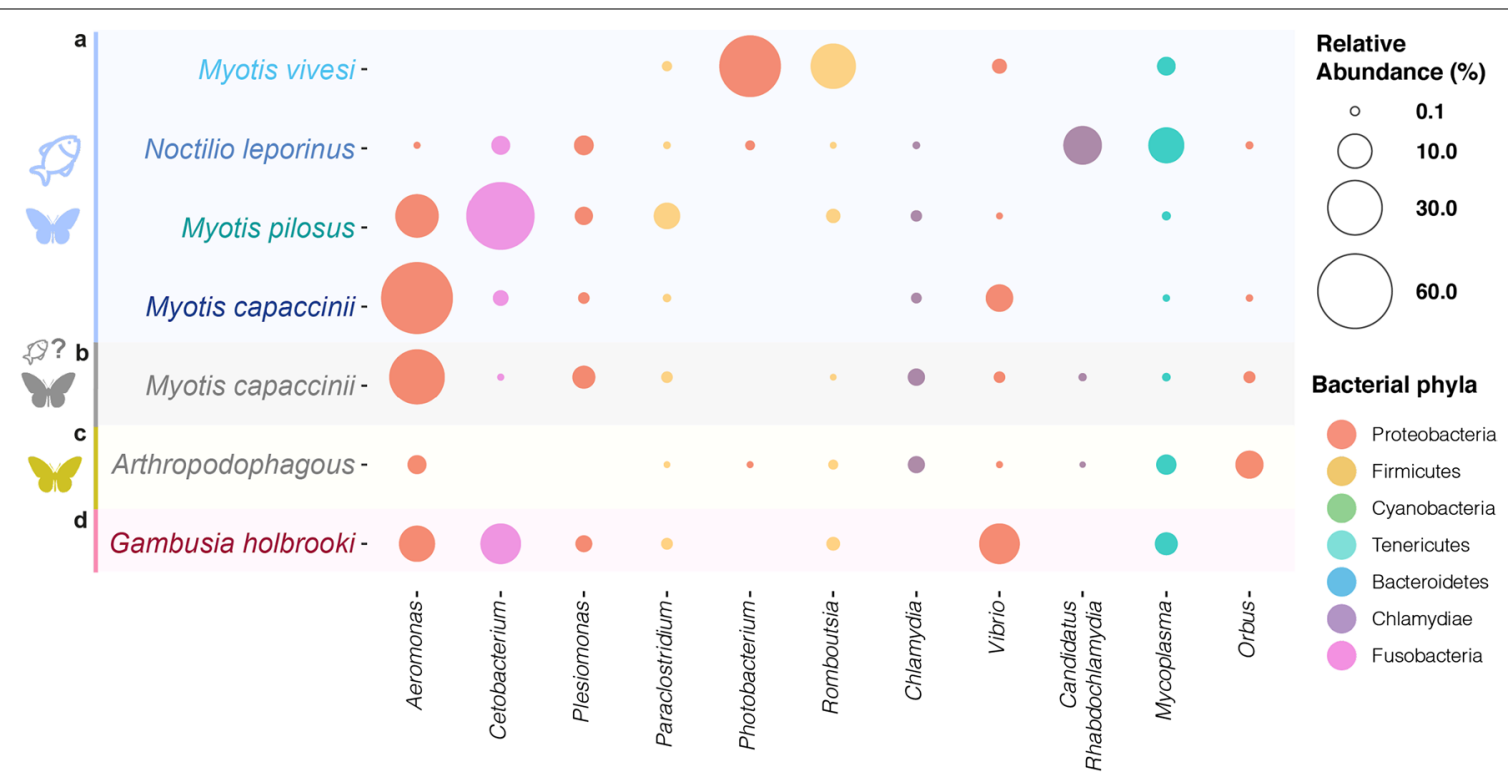

Fig. 4 Relative abundance of the most relevant bacteria enriched in piscivorous or arthropodophagous bats in a the four piscivorous bat species, b Myotis capaccinii colonies in which no piscivory has been recorded so far, $\mathbf{c}$ the eleven arthropodophagous bats analysed, and $\mathbf{d}$ the eastern mosquitofish (G. holbrooki) consumed by piscivorous M. capaccinii

species [42]. It can forage over water with different levels of salinity [43, 44], although in the area in which we sampled the bats predominantly forage in brackish water. Accordingly, we detected both the freshwater-related bacteria Cetobacterium as well as the seawater-related Photobacterium (Fig. 4). In addition, the 50 Myr of independent evolution of Noctilionids and Vespertilionids (to which the other three piscivorous Myotis species belong) [45] could have also introduced physiological differences that impede stronger convergent processes.

\section{Characteristic microbiotas of piscivorous bats are not limited to actively fishing individuals}

We assessed whether the piscivory-type gut microbiota is restricted to animals actively consuming fish, or instead extended across populations within a piscivorous species regardless of whether they commonly consume fish or not. To do so, we increased the scope of our analyses in one of the piscivorous bat species, M. capaccinii, to compare the gut microbiota of individuals from a well-known piscivorous colony [29] to those sampled at three other colonies in which no evidence of piscivory was detected after screening of bulk guano samples for fish remains, and mining shotgun metagenomic data for fish DNA traces (Additional file 1: Table S1.6).

We found that all piscivory-related bacteria enriched in $M$. capaccinii from the piscivorous colony were also enriched in all individuals from other (no known piscivory) colonies. The permutational multivariate analysis of variance also indicated that there was no difference between the gut microbiota composition of the piscivorous and allegedly non-piscivorous $M$. capaccinii (pairwise PERMANOVA ${ }_{\mathrm{U} 12}, \mathrm{R}^{2}=0.065, p$ value $_{\mathrm{FDR}}=0.090$, see Additional file 1: Fig. S1.4 and Table S1.5). Similarly, the machine learning modelling classified the gut microbiota of all M. capaccinii individuals, except one, as communities characteristic of piscivorous bats (Fig. 3d). The fact that almost all $M$. capaccinii exhibit a piscivorouslike microbiota could be indicative of a more widespread consumption of fish than previously thought. However, the screening of faecal material from multiple $M$. capaccinii colonies, through visual inspection of the faecal pellets produced by the analysed individuals and the shotgun-sequencing based DNA analysis (Additional file 1: Table S1.6), showed no traces of piscivory in any of the non-piscivory colonies. An alternative hypothesis could be that this characteristic gut microbiota is the result of an ancestral establishment of piscivory-related bacteria in the gut of this bat species as a remnant of a more widespread fishing behavior in the past.

\section{The characteristic bacteria of piscivorous bats were likely} ancestrally acquired from their fish prey

To gain further insight into the relationship between piscivorous bats and their characteristic gut bacteria, we explored the means of acquisition of piscivory-associated bacteria. The bacterial taxa enriched in piscivorous bats are common bacterial colonisers in the intestinal tract 
of freshwater and/or marine fish [46-50]. This led us to hypothesise that these taxa could have been acquired from their fish prey. To explore this, we analysed in detail the relationship between microorganisms found in the gut of $M$. capaccinii and their fish prey. For these analyses we also included as contrast arthropodophagous bats that roost in the same cave as the piscivorous $M$. capaccinii, namely Miniopterus schreibersii and Myotis myotis. We found that only two of the 28 bacteria genera overrepresented in these two species were also present in the gut environment of Gambusia, namely Mycoplasma and Desulfovibrio. Mycoplasma are common bacteria among arthropodophagous bats $[51,52]$ and Desulfovibrio inhabit the intestinal tract of many insects [53], which both bats and Gambusia prey on [54]. In contrast, we observed that all but one of microbial genera overrepresented in piscivorous $M$. capaccinii (the exception being Alysiella (Proteobacteria), see Additional file 1: Fig. S1.5) also belong to the gut microbiota of the fish species that this bat colony consumes, i.e. Gambusia holbrooki [29]. This led us to conclude that the origin of the bacteria taxa characteristic of piscivorous bats is consistent with acquisition from their fish prey.

To explore whether there is a selective mechanism in $M$. capaccinii that determines which of the bacteria acquired from the fish are established in their gut, we compared ASVs identified in M. capaccinii with the ASVs found in G. holbrooki. We found exact matches of 343 of the ASVs detected in $M$. capaccinii within the intestine of the fish species consumed. The cumulative relative representation of these ASVs was considerably higher in $M$. capaccinii $(68.60 \pm 26.4 \%$ piscivorous $M$. capaccinii, $44.8 \pm 30.80 \%$ non-piscivorous $M$. capaccinii) than among arthropodophagous bats $\left(5.14 \pm 10.30 \%\right.$; K-W $\mathrm{WM}_{\mathrm{FM}}$ cap-Arthrop: $\mathrm{X}^{2}=18.767, \mathrm{df}=3, p$ value $=0.0003$, Fig. 5a). These results suggest that many bacteria acquired from fish are not transient taxa that are only detected following recent fish consumption, but there is a filtering mechanism that determines which bacteria are established and actively maintained in the bat intestinal tracts. A similar pattern has also been observed in vultures, where the gut microbiota is conserved between captive-bred and wild individuals despite having different diets [55]. The differences between the relative representation of bacteria in the Gambusia and $M$. capaccinii intestinal tracts also support such a selective acquisition of microorganisms. The most abundant - yet most likely transitorymicrobial taxon in Gambusia, namely the cyanobacteria Oscillatoria, was not detected in the gut of M. capaccinii, and the representation of another of the most abundant taxa in Gambusia, namely Mycoplasma, was marginal in piscivorous bats. In contrast, the representation of Aeromonas was five times larger in piscivorous bats than in
Gambusia (Fig. 5b). Furthermore, the bacterial replication rate estimates from shotgun metagenomic data indicated that Aeromonas are actively replicating in the bats' gut, with no significant differences between piscivorous and non-piscivorous $M$. capaccinii (Additional file 1: Table S1.6). Aeromonas were not only found to be highly abundant and actively replicating in the intestine of $M$. capaccinii, but we also detected an extreme diversity. After studying a similar number of bat and fish individuals with the exact same methodology, the number of Aeromonas ASVs detected among piscivorous bats was ten times higher than in Gambusia (Fig. 5c). This points to the presence of multiple Aeromonas strains in bat intestines that are currently absent, or are very uncommon, in Gambusia populations around the piscivorous $M$. capaccinii colony. These could have been acquired from chironomids [56], which are the most consumed arthropod taxon by M. capaccinii [57], as Aeromonas are common gut bacteria of these arthropods [56]. However, this would not explain the differences observed between $M$. capaccinii and $M$. daubentonii, as the latter also heavily consumes chironomids [58] (Fig. 5a). Thus, the observations support the aforementioned hypothesis whereby Aeromonas would have been ancestrally acquired from fish and transferred across colonies and generations while accumulating genetic variation.

\section{Myotis capaccinii are not negatively affected by enterotoxic Aeromonas}

The high incidence and abundance of Aeromonas among piscivorous bats is striking, because most Aeromonas strains contain virulence genes responsible for encoding enterotoxins that produce gastrointestinal diseases in fish and humans [59]. To gain insights into the potential pathogenicity of Aeromonas in the bat's guts, we screened for the presence of genes encoding for enterotoxins in the metagenomic assembly. We detected the presence of the virulence gene ash3 encoding for the toxin aerolysin with a three amino acid difference to the curated reference sequence of the gene characterised from Aeromonas salmonicida (UniProKBt/Swiss-Prot Q08676). The average depth of coverage for this gene was similar to the average depth for Aeromonas $(\mathrm{t}$-test paired $\mathrm{t}=0.096, \mathrm{df}=45, p$ value $=0.92$; Fig. $5 \mathrm{~d}$ ), which indicates that the most abundant Aeromonas strains in the bat microbiota carry the gene encoding for the enterotoxin aerolysin. This toxin has the capacity to bind to eukaryotic cells and aggregate to form pores in the cell membrane leading to osmolysis, and it has also been shown to facilitate the invasion of more Aeromonas [60]. The high abundances of Aeromonas bacteria in the gut of $M$. capaccinii could a priori indicate such a pathogenic scenario, although the diversity and prevalence of Aeromonas, as well as the fact that 


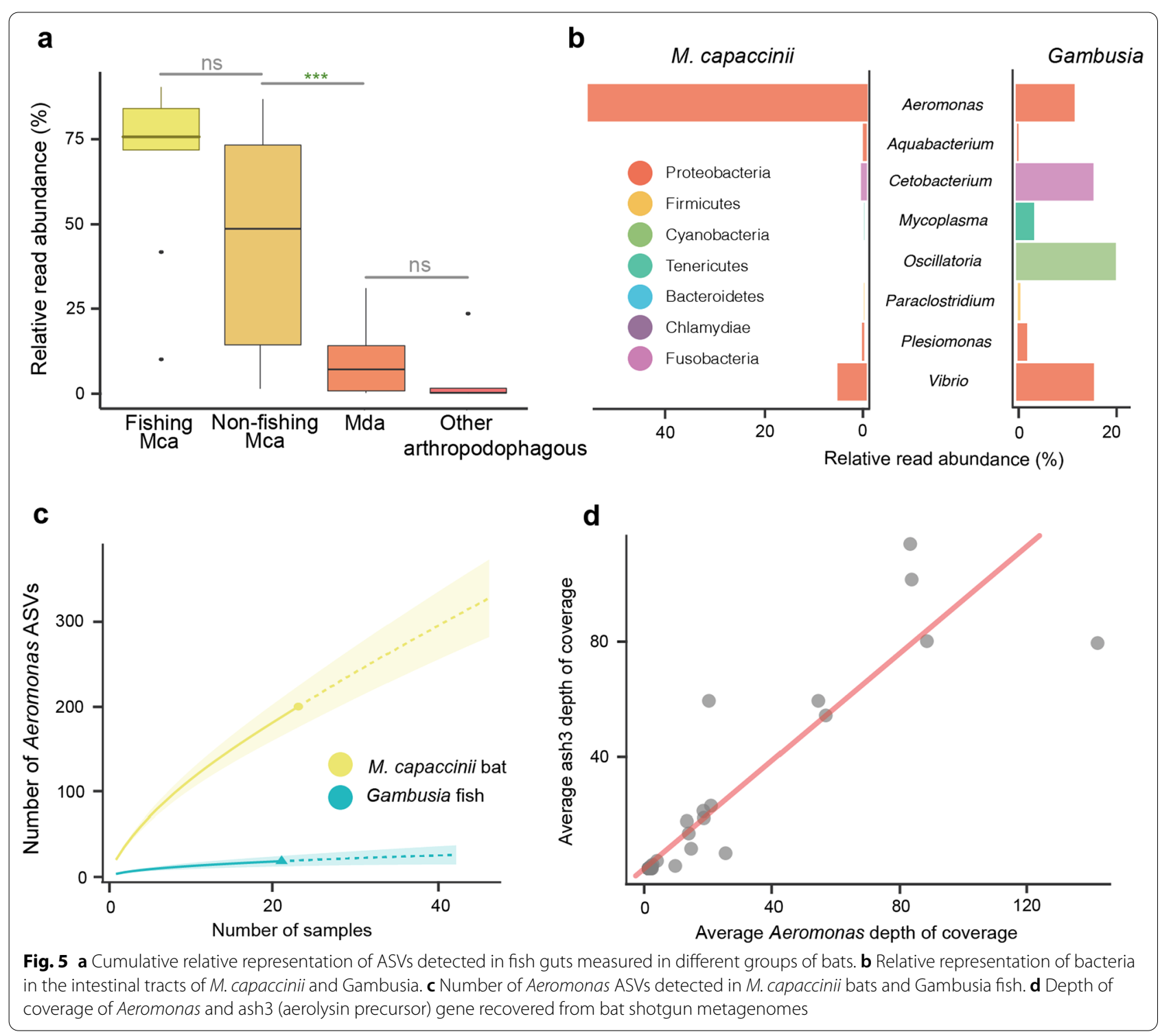

all captured animals showed no external signatures of sickness (e.g. no diarrhea, good fur appearance, normal behaviour), suggests that either Aeromonas do not heavily express the ash 3 gene in the bat gut, bats have mechanisms to protect their intestinal walls against aerolysin [61] or others members of the microbiota are acting to suppress or modulate the expression of the virulence gene of Aeromonas [62].

\section{Do piscivory-related bacteria provide nutritional benefits to bats?}

To better understand the role of piscivory-related bacteria in the gut of piscivorous bats, we explored whether the characteristic gut microbiotas exhibited by piscivorous bats might provide nutritional benefits to their hosts. To do this we implemented a dual approach consisting of (1) a Piphillin-based functional prediction [63] using the entire amplicon sequencing dataset comprising all piscivorous and arthropodophagous species, and a (2) direct shotgun sequencing-based functional profiling in a subset of piscivorous (M. capaccinii) and arthropodophagous bats.

The amplicon-based inference highlighted the enrichment of multiple pathways involved in the biosynthesis of vitamins (Fig. 6), which was also partly supported by the shotgun approach, and has been also reported in another dietary transition among bats, namely adaptation to sanguivory in vampire bats [14]. Piphillin reported an overall enrichment of pathways involved in the metabolism of vitamins B2, B6 and B9 in piscivorous bats. Enrichment 


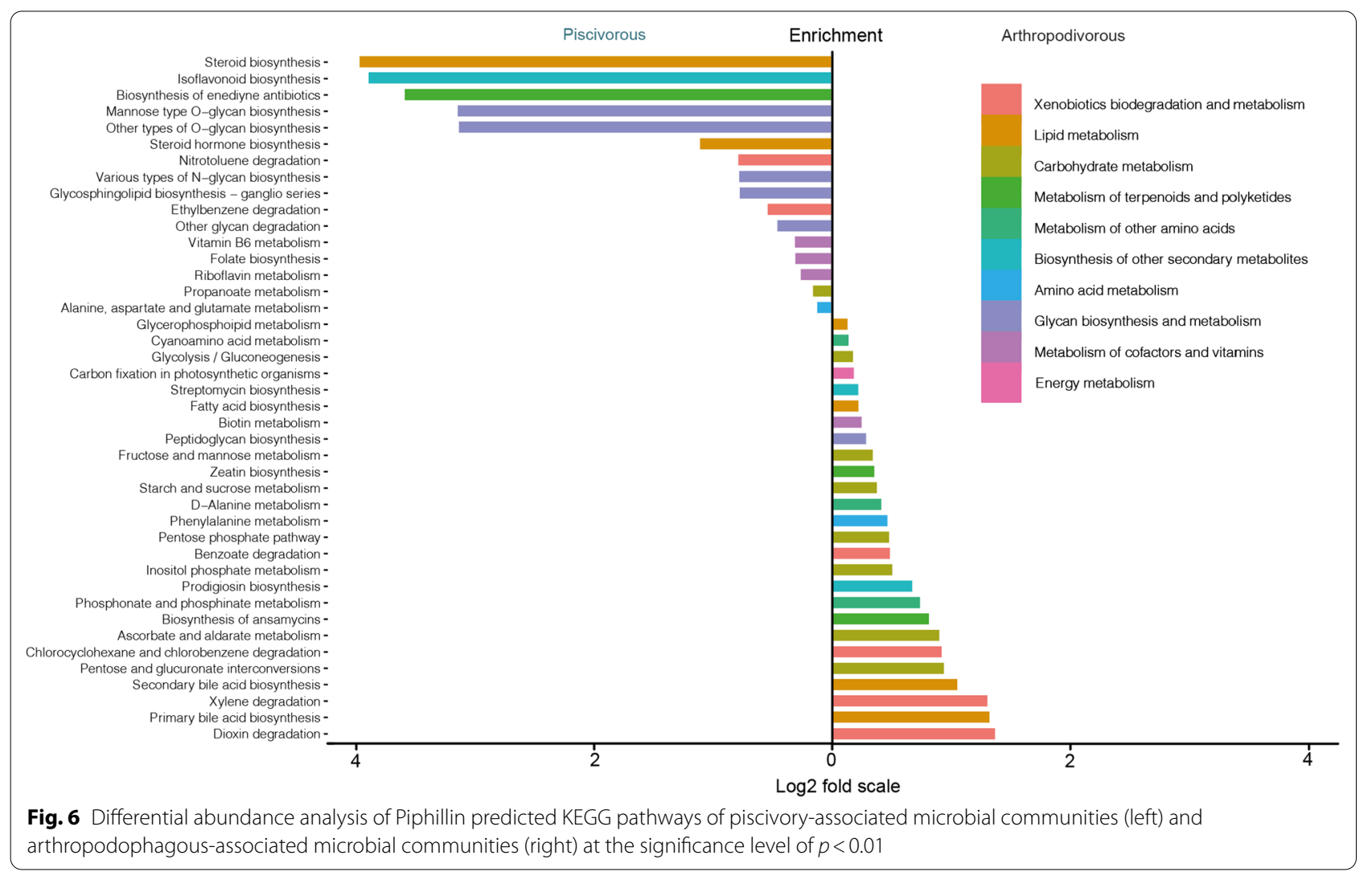

of biosynthesis of vitamins B6 and B9 was not supported by the $M$. capaccinii shotgun data $\left(\mathrm{t}\right.$-test ${ }_{\mathrm{B} 6}: \mathrm{t}=1.32$, $\mathrm{df}=12.43, p$ value $=0.209 ; \mathrm{t}_{\text {-test }} \mathrm{B}_{\mathrm{B}}: \mathrm{t}=0.270, \mathrm{df}=8.79$, $p$ value $=0.793)$. However, we found that enrichment of genes involved in the related vitamin B2 biosynthesis was borderline significant $\left(\mathrm{t}\right.$-test $\mathrm{B}_{\mathrm{B} 2}: \mathrm{t}=2.07, \mathrm{df}=14.65$, $p$ value $=0.056$ ), and $\mathrm{B} 12$ biosynthesis was significantly enriched in $M$. capaccinii $\left(\mathrm{t}\right.$-test ${ }_{\mathrm{B} 12}: \mathrm{t}=3.40, \mathrm{df}=11.75$, $p$ value $=0.005$ ). The most abundant genes involved in vitamin B12 biosynthesis were assigned to Aeromonas, which is the most abundant taxon among $M$. capaccinii. The incidence of Aeromonas in M. pilosus is much lower, yet they exhibit an increased abundance of Cetobacterium, which are also known to produce vitamin B12 [64]. These results suggest that the microbiota of piscivorous bats holds the capacity to synthesize a range of vitamins that contribute to various metabolic and physiological processes.

The analysis of the shotgun sequence data also yielded the enrichment of a number of other metabolic functions observed in the Piphillin-based predictive approach, among which glycan metabolism stood out, with five pathways enriched in piscivorous bats. Glycans are known to play a central role in shaping the composition and activity of intestinal microorganisms, and fish are among the richest sources of certain types of glycans such as glycosphingolipids [65], whose metabolism was also enriched in piscivorous bats. We also detected enrichment of a number of pathways related with lipid metabolism, including alpha-linolenic acid (ALA) and fatty acid degradation that are involved in metabolising omega-3 fatty acids [66]. These types of compounds are highly represented in fish flesh, and they are well-known for their anti-inflammatory activity as rich sources of long-chain fatty acids that are metabolised into shortchain fatty acids (SCFAs) [67].

\section{Conclusions}

Our analysis of the faecal microbiota of the four piscivorous bat species, eleven arthropodophagous counterparts and one fish species consumed by one of the piscivorous bats, enabled us to disentangle the taxonomic and functional microbiota features associated with fishing behaviour in bats. Our results indicate that piscivorous bats carry a microbiota that is distinct to that of arthropodophagous bats. The characteristic bacteria of piscivorous bats likely provide nutritional benefits to their hosts, by synthesizing essential compounds and facilitating the metabolism of complex carbohydrates and lipids acquired from fish and arthropods. 
The fact that these features are most strongly represented in actively piscivorous bats, yet also present in non-piscivorous colonies of piscivorous bat species, while almost completely absent in arthropodophagous bats, suggests that piscivory-like microbial communities are not a recently acquired trait. In contrast, microbiota fingerprints suggest that the trophic niche expansion produced by incorporating fish into the diet enabled bats to acquire beneficial bacteria otherwise largely inaccessible in the terrestrial environment, which have been established and spread across populations. All in all, our results point to an active contribution of microorganisms to facilitating dietary shifts in vertebrates.

\section{Methods}

\section{Sampling and sample storage}

We captured 98 wild individuals of 15 bat species in 22 localities across Europe, China, Israel, Mexico, and Costa Rica using harp traps and mist nets (see Additional file 1: Table S1.1). The sampled species were Eptesicus bottae (Ebo), Hypsugo ariel (Har), Miniopterus schreibersii (Msc), Myotis capaccinii (Mca), M. daubentonii (Mda), M. emarginatus (Mem), M. myotis (Mmy), M. pilosus (Mpi), M. vivesi (Mvi), Noctilio leporinus (Nle), Pipistrellus kuhlii (Pku), Rhinolophus blasii (Rbl), R. euryale (Reu), $R$. hipposideros (Rhi) and $R$. ferrumequinum (Rfe). To avoid sample cross-contamination, each bat was kept separately in a clean, single-use cotton bag for 15-20 min, then identified, sexed and aged before releasing them. Faecal pellets were collected from the bags and stored in $1.5 \mathrm{ml}$ collection tubes filled with ethanol.

For the analysis of fish microbiota, 13 individuals of the exotic fish Gambusia holbrooki were captured using minnow trap nets at four wetlands in south-eastern Spain, where the piscivorous colony of Myotis capaccinii is located. Fish were kept in containers with water from the sample location, which were continually oxygenated to prevent hypoxia. Within a maximum period of four hours after sampling, fish were euthanized by a quick blow to the head in compliance with the Spanish law on animal research ethics (RD 53/2013) and the European Directive on the protection of animals used for scientific purposes (2010/63/EU). The entire gastrointestinal tract was removed from the fish using scalpels, after which the gut content was separated from the intestine and stored in $1.5 \mathrm{ml}$ collection tubes filled with ethanol.

All samples were refrigerated $\left(4-8^{\circ} \mathrm{C}\right)$ until they were transported to the laboratory, after which they were stored at $-20^{\circ} \mathrm{C}$ until DNA extraction. All captures were authorised by the competent authorities of the countries in which they were carried out.

\section{Morphological analysis of bulk guano samples}

Bulk faecal material was collected from the roosting caves of M. capaccinii. In the laboratory each bulk was homogenized with water and then filtered through two laboratory sieves with different mesh sizes $(2 \mathrm{~mm}$ and $0.5 \mathrm{~mm}$ ). The unfiltered material was inspected by magnifying lens for fish remains, such as otoliths, bones and scales.

\section{Laboratory work \\ DNA extraction}

DNA was extracted in a dedicated pre-PCR laboratory following a randomised setup from 1-3 bat droppings (ca. $20 \mathrm{mg}$ ) of each bat individual and the entire gut content of the fish. The PowerSoil ${ }^{\circledR}$ DNA Isolation Kit (MoBio, CA, USA) was used following the manufacturer's protocol (2016 version) with the modifications explained in Alberdi et al. [68]. Each extraction round included 23 samples and one negative extraction control. The final DNA extracts $(50 \mu \mathrm{l})$ were aliquoted in five subsamples to avoid any potential contamination of the entire volume during later processing.

\section{5 amplicon sequencing}

The V3 and V4 hypervariable regions of the bacterial $16 \mathrm{~S}$ rRNA were targeted using the $341 \mathrm{~F} / 806 \mathrm{R}$ primer pair $[69$, 70]. Prior to the tagged PCRs, the most optimal annealing temperature was assessed by amplifying five extracts and one extraction blank using temperatures ranging from $50^{\circ} \mathrm{C}$ to $62^{\circ} \mathrm{C}$. Additionally, quantitative PCR (qPCR) screening was carried out with multiple DNA template volumes on six extracts and both extraction blanks to (1) assess contamination of extraction blanks, (2) determine the optimal cycle number for the subsequent PCRs, and (3) estimate the maximum template amount for the following tagged PCR amplifications in which PCR inhibitory substances, copurified with the DNA, would not distort the amplification [71-73]. PCRs were run on an Applied Biosystems 2720 Thermal Cycler with a reaction volume of $25 \mu \mathrm{l}$. Each PCR reaction contained $2.5 \mu \mathrm{l}$ of AmpliTaq Gold buffer (final concentration 1X), $2.5 \mu \mathrm{l}$ of $\mathrm{MgCl}_{2}(2.5 \mathrm{mM}), 1.5 \mu \mathrm{l}$ of BSA $(1.2 \mathrm{ng} / \mu \mathrm{l}), 0.5 \mu \mathrm{l}$ of dNTP $(0.2 \mathrm{mM}), 0.5 \mu \mathrm{l}$ of AmpliTaq Gold DNA polymerase $(0.1$ $\mathrm{U} / \mu \mathrm{l}), 2 \mu \mathrm{l}$ of primer mix $(0.8 \mathrm{mM})$ and $13.5 \mu \mathrm{l}$ of $\mathrm{ddH}_{2} \mathrm{O}$. PCR settings were $95^{\circ} \mathrm{C}$ for $10 \mathrm{~min}, 30$ cycles of $95^{\circ} \mathrm{C}$ for $15 \mathrm{~s}, 53^{\circ} \mathrm{C}$ for $20 \mathrm{~s}$ and $72^{\circ} \mathrm{C}$ for $40 \mathrm{~s}$ and at last $72^{\circ} \mathrm{C}$ for $10 \mathrm{~min}$. The PCR products from different samples were pooled in batches of 24 samples while making sure that each sample had its own unique tagged primer set within the pool, in order to enable tracking the sample back to the individual of origin [74]. All amplicon pools were subsequently purified at 1:1 beads:DNA volume-ratio 
to remove non-target DNA and primer dimers using SPRI beads $[75,76]$. Sequencing libraries were prepared using the Tagsteady library building protocol [77], and sequenced on an Illumina MiSeq machine using 250PE chemistry.

\section{Shotgun sequencing}

A subset of DNA extracts was also processed for shotgun sequencing. Specifically, 18 samples belonging to 7 arthropodophagous and 11 piscivorous bat individuals were sonicated into fragment-lengths around $350 \mathrm{bp}$ on a Covaris S220x Focused ultrasonicator (Covaris Inc., Woburn, MA). Fragmented DNA (200 ng) was built into shotgun sequencing libraries using the BEST protocol, as described in [78]. Libraries were purified using SPRI beads (1:1.4 DNA:beads ratio) followed by a qPCR screening to determine the optimal number of cycles for PCR indexing with different reverse indices. Indexing cycle numbers adjusted to the molarity of each library were in the range of 7-16. After indexing, an additional purification step was performed using SPRI beads (1:1.2 DNA:beads ratio) before pooling multiple libraries for multiplex sequencing in an Illumina HiSeq2500 platform with 150PE chemistry.

\section{Data analysis}

\section{Bioinformatic processing of amplicon data}

Amplicon sequencing reads were demultiplexed based on library indices using AdapterRemoval [79]. As the library building approach we used is based on adapter ligation rather than PCR amplification, the resulting DNA sequences can be either in Forward-Reverse or Reverse-Forward direction. Using Cutadapt 1.18 [80], we identified primer locations, and reverse complemented the reads in Reverse-Forward direction to ensure unidirectionality of all sequences. Taxonomic assignment was done by the naive Bayesian classifier method with default settings as implemented in DADA2 [81] in R 3.6.1 [82], against SILVA 16S rRNA gene reference taxonomy database. The initial ASV table was generated for the 15,856 ASVs that were taxonomically annotated.

Contamination filtering was independently performed in each batch of samples with its corresponding controls using decontam (Davis et al., 2018) [99]. The 18 putative contaminants were removed from the ASV table. Rarefaction curves were plotted using the $R$ package vegan to identify samples not reaching a diversity saturation plateau in the number of sequencing reads. Based on this analysis, six samples with fewer than 10,000 reads were removed from the dataset. To minimise impact of potential false positives, ASVs with less copies than $0.01 \%$ of the total number of reads of each sample were removed, which reduced the ASV table to 9298 ASVs. Finally, the
ASVs that could not be taxonomically identified as Bacteria (129 ASVs) or to Phylum and Class level (37 ASVs) were removed and the output ASV table (9132 ASVs) was used for downstream analyses.

\section{Functional prediction from amplicon data}

Functional prediction of the microbial content from $16 \mathrm{~S}$ rRNA gene sequencing data was performed using Piphillin [63]. Piphillin predicts metagenomic content via direct nearest-neighbor matching between 16S rRNA gene amplicons and genomes from KEGG reference database. The analysis was run using a 99\% ID cutoff [83]. Only metabolic pathways were included in the differential abundance analysis. The differential abundance analysis in the functionality of the gut microbiota of arthropodophagous and piscivorous bats was performed using the univariate DESeq2 method [84]. DESeq2 was based on the negative binomial Wald test and parametric fitType. Only ASVs with False Discovery Rate adjusted $p$ value $<0.01$ were considered statistically significant. ggplot() function of the R package tidyverse was used for data visualization [85].

\section{Ensemble machine learning-based modelling}

We implemented an ensemble approach consisting of three machine learning algorithms, namely logistic regression, Random Forest and XGBoost, to first gain further insights into the compositional differences of the gut microbiota of arthropodophagous and piscivorous bats, and second, to ascertain the classification into arthropodophagous-like or piscivorous-like microbiotas in the three $M$. capaccinii populations with "uncertain" dietary preferences. We used a set of 84 samples (47 arthropodophagus and 37 piscivorous) for training the models using the caretList function in the R library caretEnsemble $[19,86]$. ROC was used to select the optimal model using the largest value, and arthropodophagus was used as the 'positive' class to compute sensitivity (Sens) and specificity (Spec) values [87]. We used caretStack to merge the models into a predictive meta-model. Feature (ASV) importance for the classification using the meta-model was obtained using the function varImp. The ensemble model was used to predict the classification of $15 \mathrm{M}$. capacinii from allegedly non-piscivorous colonies using the function predict, after which prediction statistics were obtained using the confusionMatrix and custom functions.

\section{Diversity and compositional analysis}

All the analyses were carried out in the $\mathrm{R}$ statistical environment [82] and the diversity analyses were performed based on the relative abundance of each ASV (calculated as ASV read depth over total read depth 
per library). Diversity analyses and visualization were carried out using the div_test and div_test_plot function of the $\mathrm{R}$ package hilldiv based on abundancebased Hill numbers [18]. The maximum likelihood phylogeny generated from ASV sequences using RAxML-NG was employed for phylogenetic diversity metrics. Richness was computed as the neutral Hill number of order of diversity $q=0$; richness and evenness was computed as the neutral Hill number of order of diversity $\mathrm{q}=1-$ i.e. Shannon diversityand richness, evenness and regularity was computed as the phylogenetic Hill number of order of diversity $\mathrm{q}=1$ [88]. Diversity comparisons between species were carried out using the Kruskal-Wallis (K-W) rank sum test, followed by a posthoc Dunn's test with Bonferroni-corrected $p$ values. The Jaccard-type turnovercomplement ( $\mathrm{SqN})$ was computed using beta_dis() function of package hilldiv. The function was run by inputting values from the object outputted by the div part() function.

Compositional differences were computed by pairwise distances among samples using the pair_dis function of the R package hilldiv based on abundancebased Hill numbers [18]. Compositional differences were contrasted using permutational multivariate analyses of variance (PERMANOVA) with 999 permutations using the vegan::adonis function [89]. calc_pairwise_permanovas function of $\mathrm{R}$ package mctoolsr was used to calculate pairwise post-hoc comparisons between samples [90]. The level of homogeneity of dispersion within groups was first analysed using vegan::betadisper and vegan::permutest function ( $p$ value $>0.05$, see Additional file 1: Table S1.4) [89]. Since this assumption was not met when richness was computed, in the results section only NMDS and PERMANOVA based on pairwise distances calculated through the neutral Hill number of order of diversity $q=1$ (PERMANOVA ${ }_{U 12}$ ) and phylogenetic Hill number of order of diversity $q=1$ (PERMANOVA ${ }_{U 12}$ ) are shown. Statistically significant results were considered at $p$ values $<0.05$, which were adjusted for False discovery rate ( $p$ value $_{\mathrm{FDR}}$ ) (Benjamini and Hochberg 1995) [100]. The dis_nmds function of package hilldiv was used for plotting the NMDSs. The core microbiota was explored using the microbiome package [91]. The detection prevalence of $90 \%$ across samples was setted up. We analysed the core microbiota of all studied bats together as well as arthropodophagous and piscivorous bat species separately. A differential abundance analysis using the univariate DESeq2 method [84] was performed to identify the individual ASVs driving the changes between arthropodophagous and piscivorous bats.

\section{Bioinformatic processing of shotgun data}

Shotgun sequencing reads were demultiplexed and quality-filtered using AdapterRemoval. Duplicated reads were filtered using seqkit 0.7.1 [92] and bat and fish DNA was removed from the dataset using bwa mem [93] by mapping the reads against the reference genome sequences of the closest relative of Myotis capaccinii, namely M. myotis and the draft genome of Gambusia holbrooki, respectively. Presence of fish remains in metagenomic data was assessed through analysing the mapping rate to the $G$. holbrooki genome. Due to conserved genomic regions across vertebrates, mapping rate to fish genomes even in the absence of fish remains is not zero, but oscillates at a basal level of a few sequences per million reads. Hence, the threshold we employed to consider fish DNA remains were actually detected was three standard deviations larger than the average mapping rate of arthropodophagous bats to the G. holbrooki genome. Subsequently, we mapped the preprocessed reads against the reference genomes of Aeromonas veronii (ASM869370v1) and used the software iRep to estimate bacterial replication rates by means of the peak-to-trough ratio (PTR) [94, 95]. Metagenomic reads were then co-assembled using Megahit [96] and open reading frames (ORF) predicted using Prodigal 2.6.3 [97]. ORFs were functionally annotated by aligning them against the KEGG database using GhostKoala [98], and the reads of each sample mapped to the ORF catalogue to obtain the overall functional profile of the microbial metagenome. To analyse the presence of enterotoxin-encoding genes, we aligned the ORF sequences to a custom reference database of 86 amino acid sequences of Aeromonas enterotoxic genes created using sequences available at Uniprot. Then, reads of each sample were mapped to the Aeromonas enterotoxic genes to obtain the depth of coverage of such genes. Statistical comparisons were made with Student t-test in R.

\section{Supplementary Information}

The online version contains supplementary material available at https://doi. org/10.1186/s42523-021-00137-w.

Additional file 1. Supplementary figures and tables.

\section{Acknowledgements}

The authors thank the staff at the Danish National High-Throughput DNA Sequencing Centre for generating the sequencing data. We are also grateful to all the authorities and institutions from Spain, Mexico, China, Israel and Costa Rica that issued the permits for bat sampling, and specially to the Regional Government of Valencia and the personnel from El Palmar research center for facilitating bat and fish sampling. We are grateful as well to the Prescott College Kino Bay Center, the Area de Protección de Flora y Fauna Islas del Golfo de California-Baja California, Lorayne Meltzer, Martin Ziebell, Leonel Moreno, and Vaporrúb, which provided logistic support during our work in Mexico. 


\section{Authors' contributions}

OA, AA and MTPG designed the study. OA, AA, LN, EM, GC, JJF-M. and AL participated in the sample and data collection. OA, LN, EM, and AA performed the laboratory procedures. OA and LN performed the DNA analyses, and OA and AA the statistical analysis. OA and AA wrote the manuscript. All authors contributed to and approved the final version.

\section{Funding}

The authors thank the following for funding their research: The Carlsberg Foundation's Distinguished Postdoctoral Fellowship to O.A. (CF15-0619), The Danish National Research Foundation award to M.T.P.G. (DNRF143), Villum Fonden Grant to M.T.P.G. (17417), Lundbeckfonden Grant to A.A. (R2502017-1351), Danish Council for Independent Research Grants to A.A. (DFF 5051-00033), Grant from Consejo Nacional de Ciencia y Tecnología to L.G.H.M. (237774).

\section{Availability of data and materials}

The raw data have been deposited at the NCBI SRA database under the project accession number PRJEB47836. Bioinformatics pipelines used to process the sequence data and generate count tables are available at https://github. com/ostaizka/fishing-bats.

\section{Declarations}

\section{Ethics approval and consent to participate}

Not applicable.

\section{Consent for publication}

Not applicable.

\section{Competing interests}

The authors declare that they have no competing interests.

\section{Author details}

${ }^{1}$ Center for Evolutionary Hologenomics, GLOBE Institute, University of Copenhagen, 1353 Copenhagen, Denmark. ${ }^{2}$ University of Exeter, Streatham Campus, Biosciences, Exeter EX4 4PS, UK. ${ }^{3}$ Sede del Sur, Universidad de Costa Rica, \#4000 Alamedas, Golfito 60701, Costa Rica. ${ }^{4}$ Smithsonian Tropical Research Institute, Apartado 0843-03092 Balboa, Ancón, República de Panamá. ${ }^{5}$ Estación de Biología Chamela, Instituto de Biología, Universidad Nacional Autónoma de México, Apartado Postal 21, San Patricio, 48980 Jalisco, Mexico. ${ }^{6}$ Laboratorio de Sistemas de Información Geográfica, Departamento de Zoología, Instituto de Biología, Universidad Nacional Autónoma de México, Circuito Exterior s/n, Ciudad Universitaria, 04510 Mexico City, Mexico. ${ }^{7}$ Jilin Provincial Key Laboratory of Animal Resource Conservation and Utilization, Northeast Normal University, Changchun 130117, China. ${ }^{8}$ University Museum, Norwegian University of Science and Technology, 7491 Trondheim, Norway.

Received: 10 February 2021 Accepted: 4 October 2021

Published online: 28 October 2021

\section{References}

1. Perry GH, Dominy NJ, Claw KG, Lee AS, Fiegler H, Redon R, et al. Diet and the evolution of human amylase gene copy number variation. Nat Genet. 2007:39:1256-60.

2. Price SA, Hopkins SSB. The macroevolutionary relationship between diet and body mass across mammals. Biol J Linn Soc Lond. 2015;115:173-84.

3. Karasov WH, Martínez del Rio C, Caviedes-Vidal E. Ecological physiology of diet and digestive systems. Annu Rev Physiol. 2011;73:69-93.

4. Baldo L, Riera JL, Tooming-Klunderud A, Albà MM, Salzburger W. Gut microbiota dynamics during dietary shift in Eastern African cichlid fishes. PLOS ONE. 2015;10:e0127462.

5. Hao YT, Wu SG, Xiong F, Tran NT, Jakovlić I, Zou H, et al. Succession and fermentation products of grass carp (Ctenopharyngodon idellus) hindgut microbiota in response to an extreme dietary shift. Front Microbiol. 2017;8:1585.
6. Flint HJ, Scott KP, Duncan SH, Louis P, Forano E. Microbial degradation of complex carbohydrates in the gut. Gut Microbes. 2012;3:289-306.

7. Nicholson JK, Holmes E, Kinross J, Burcelin R, Gibson G, Jia W, et al. Hostgut microbiota metabolic interactions. Science. 2012;336:1262-7.

8. Nelson T. Factors Influencing the Gut Microbiota of Antarctic Seals. Doc tor of Philosophy. School of Biological, Earth \& Environmental Sciences; 2012.

9. Bredon M, Dittmer J, Noël C, Moumen B, Bouchon D. Lignocellulose degradation at the holobiont level: teamwork in a keystone soil invertebrate. Microbiome. 2018;6:162.

10. Fenton MB, Simmons NB. Bats: a world of science and mystery. Chicago: University of Chicago Press; 2014.

11. Wang K, Tian S, Galindo-González J, Dávalos LM, Zhang Y, Zhao H. Molecular adaptation and convergent evolution of frugivory in Old World and neotropical fruit bats. Mol Ecol. 2020;29:4366-81.

12. Ingala MR, Simmons NB, Perkins SL. Bats are an untapped system for understanding microbiome evolution in mammals. mSphere. 2018. https://doi.org/10.1128/mSphere.00397-18.

13. Price ER, Brun A, Caviedes-Vidal E, Karasov WH. Digestive adaptations of aerial lifestyles. Physiology. 2015;30:69-78.

14. Zepeda Mendoza ML, Xiong Z, Escalera-Zamudio M, Runge AK, Thézé J, Streicker D, et al. Hologenomic adaptations underlying the evolution of sanguivory in the common vampire bat. Nat Ecol Evol. 2018;2:659-68.

15. Aizpurua O, Alberdi A. Ecology and evolutionary biology of fishing bats. Mamm Rev. 2018:48:284-97.

16. Nogales-Mérida S, Gobbi P, Józefiak D, Mazurkiewicz J, Dudek K, Rawski $M$, et al. Insect meals in fish nutrition. Rev Aquacult. 2019:11:1080-103.

17. Segura-Trujillo CA. Letter to the editor. Arthropodophagy vs "insectivory" in bats. Therya. 2017;8:89-90.

18. Alberdi A, Gilbert MTP. hilldiv: an R package for the integral analysis of diversity based on Hill numbers. bioRxiv. 2019. https://doi.org/10.1101/ 545665.

19. Deane-Mayer ZA, Knowles JE. caretEnsemble: Ensembles of caret models. R package version. 2016;2.

20. Carrillo-Araujo M, Taş N, Alcántara-Hernández RJ, Gaona O, Schondube JE, Medellín RA, et al. Phyllostomid bat microbiome composition is associated to host phylogeny and feeding strategies. Front Microbiol. 2015;6:447.

21. Hughes GM, Leech J, Puechmaille SJ, Lopez JV, Teeling EC. Is there a link between aging and microbiome diversity in exceptional mammalian longevity? PeerJ. 2018;6:e4174.

22. Ingala MR, Simmons NB, Wultsch C, Krampis K, Speer KA, Perkins SL. Comparing microbiome sampling methods in a wild mammal: feca and intestinal samples record different signals of host ecology. Evol Front Microbiol. 2018;9:803.

23. Dietrich M, Kearney T, Seamark ECJ, Markotter W. The excreted microbiota of bats: evidence of niche specialisation based on multiple body habitats. FEMS Microbiol Lett. 2017. https://doi.org/10.1093/femsle/ fnw284.

24. Delsuc F, Metcalf JL, Wegener Parfrey L, Song SJ, González A, Knight R. Convergence of gut microbiomes in myrmecophagous mammals. Mol Ecol. 2014;23:1301-17.

25. Phillips CD, Hanson J, Wilkinson JE, Koenig L, Rees E, Webala P, et al. Microbiome structural and functional interactions across host dietary niche space. Integr Comp Biol. 2017;57:743-55.

26. Louca S, Polz MF, Mazel F, Albright MBN, Huber JA, O'Connor MI, et al. Function and functional redundancy in microbial systems. Nat Ecol Evol. 2018:2:936-43.

27. Stadelmann B, Lin L-K, Kunz TH, Ruedi M. Molecular phylogeny of New World Myotis (Chiroptera, Vespertilionidae) inferred from mitochondrial and nuclear DNA genes. Mol Phylogenet Evol. 2007;43:32-48.

28. Ma J, Zhang J, Liang B, Zhang L, Zhang S, Metzner W. Dietary Characteristics of Myotis ricketti in Beijing, North China. J Mammal. 2006:87:339-44.

29. Aizpurua O, Garin I, Alberdi A, Salsamendi E, Baagøe H, Aihartza J. Fishing long-fingered bats (Myotis capaccinii) prey regularly upon exotic fish. PLOS ONE. 2013;8:e80163.

30. Ma J, Jones G, Zhang S, Shen J, Metzner W, Zhang L, et al. Dietary analysis confirms that Rickett's big-footed bat (Myotis ricketti) is a piscivore. J Zool. 2003;261:245-8. 
31. Otalora-Ardila A, Herrera MLG, Flores-Martinez JJ, Voigt CC. Marine and terrestrial food sources in the diet of the fish-eating myotis (Myotis vivesi). J Mammal. 2013;94:1102-10.

32. Dehler CE, Secombes CJ, Martin SAM. Seawater transfer alters the intestinal microbiota profiles of Atlantic salmon (Salmo salar L.). Sci Rep. 2017;7:13877

33. Sullam KE, Essinger SD, Lozupone CA, O'Connor MP, Rosen GL, Knight $R$ et al. Environmental and ecological factors that shape the gut bacterial communities of fish: a meta-analysis. Mol Ecol. 2012;21:3363-78.

34. Van Horn DJ, Okie JG, Buelow HN, Gooseff MN, Barrett JE, Takacs-Vesbach CD. Soil microbial responses to increased moisture and organic resources along a salinity gradient in a polar desert. Appl Environ Microbiol. 2014:80:3034-43.

35. Pacheco-Sandoval A, Schramm Y, Heckel G, Brassea-Pérez E, MartínezPorchas M, Lago-Lestón A. The Pacific harbor seal gut microbiota in Mexico: its relationship with diet and functional inferences. PLoS ONE. 2019;14:e0221770.

36. Drinkwater R, Goodwin A, Cush J, Korstian JM, Chumchal MM, Gerardo Herrera ML, et al. Molecular diet analysis of the marine fish-eating bat, Myotis vivesi, and potential mercury exposure. J Mammal. 2020;99:752-9.

37. Semova I, Carten JD, Stombaugh J, Mackey LC, Knight R, Farber SA, et al. Microbiota regulate intestinal absorption and metabolism of fatty acids in the zebrafish. Cell Host Microbe. 2012;12:277-88.

38. Herlemann DPR, Labrenz M, Jürgens K, Bertilsson S, Waniek JJ, Andersson AF. Transitions in bacterial communities along the $2000 \mathrm{~km}$ salinity gradient of the Baltic Sea. ISME J. 2011:5:1571-9.

39. Moi IM, Roslan NN, Leow ATC, Ali MSM, Rahman RNZRA, Rahimpour A, et al. The biology and the importance of Photobacterium species. App Microbiol Biotechnol. 2017;101:4371-85.

40. Alfaro B, Hernandez I. Evolution of the indigenous microbiota in modified atmosphere packaged Atlantic horse mackerel (Trachurus trachurus) identified by conventional and molecular methods. Int J Food Microbiol. 2013;167:117-23.

41. Svanevik CS, Lunestad BT. Characterisation of the microbiota of Atlantic mackerel (Scomber scombrus). Int J Food Microbiol. 2011;151:164-70.

42. Bordignon MO. Diet of the fishing bat Noctilio leporinus (Linnaeus) (Mammalia, Chiroptera) in a mangrove area of southern Brazil. Revista Brasileira de Zoologia. 2006;23:256-60

43. Bordignon MO. Activity pattern and foraging behavior of bulldog-bat Noctilio leporinus (Linnaeus) (Chiroptera, Noctilionidae) in Guaratuba Bay, Paraná State, Brazil. Rev Bras Zool. 2006;23:50-7.

44. Schnitzler H-U, Kalko EKV, Kaipf I, Grinnell AD. Fishing and echolocation behavior of the greater bulldog bat, Noctilio leporinus, in the field. Behav Ecol Sociobiol. 1994;35:327-45.

45. Teeling EC, Springer MS, Madsen O, Bates P, O'brien SJ, Murphy WJ. A molecular phylogeny for bats illuminates biogeography and the fossil record. Science. 2005;307:580-4.

46. Nayak SK. Role of gastrointestinal microbiota in fish. Aquac Res. 2010;41:1553-73.

47. Egerton S, Culloty S, Whooley J, Stanton C, Ross RP. The gut microbiota of marine fish. Front Microbiol. 2018;9:873.

48. Tsuchiya C, Sakata T, Sugita H. Novel ecological niche of Cetobacterium somerae, an anaerobic bacterium in the intestinal tracts of freshwater fish. Lett Appl Microbiol. 2008:46:43-8.

49. van Kessel MA, Dutilh BE, Neveling K, Kwint MP, Veltman JA, Flik G, et al. Pyrosequencing of $16 \mathrm{~S}$ rRNA gene amplicons to study the microbiota in the gastrointestinal tract of carp (Cyprinus carpio L.). AMB Express. 2011;1:41.

50. Ingerslev H-C, von Gersdorff JL, Lenz Strube M, Larsen N, Dalsgaard I, Boye $M$, et al. The development of the gut microbiota in rainbow trout (Oncorhynchus mykiss) is affected by first feeding and diet type. Aquaculture. 2014;424-425:24-34.

51. Banskar S, Mourya DT, Shouche YS. Bacterial diversity indicates dietary overlap among bats of different feeding habits. Microbiol Res. 2016:182:99-108.

52. Xiao G, Liu S, Xiao Y, Zhu Y, Zhao H, Li A, et al. Seasonal changes in gut microbiota diversity and composition in the greater horseshoe bat. Front Microbiol. 2019;10:2247.

53. Arias-Cordero E, Ping L, Reichwald K, Delb H, Platzer M, Boland W. Comparative evaluation of the gut microbiota associated with the below- and above-ground life stages (larvae and beetles) of the forest cockchafer, Melolontha hippocastani. PLoS ONE. 2012;7:e51557.

54. Pyke GH. A review of the biology of Gambusia affinis and G. holbrooki. Rev Fish Biol Fish. 2005;15:339-65.

55. Roggenbuck M, Bærholm Schnell I, Blom N, Bælum J, Bertelsen MF, Sicheritz-Pontén T, et al. The microbiome of New World vultures. Nat Commun. 2014:5:5498.

56. Laviad S, Halpern M. Chironomids' relationship with Aeromonas species. Front Microbiol. 2016:7:736.

57. Almenar D, Aihartza J, Goiti U, Salsamendi E, Garin I. Diet and prey selection in the trawling long-fingered bat. J Zool. 2008;274:340-8.

58. Vesterinen EJ, Lilley T, Laine VN, Wahlberg N. Next generation sequencing of fecal DNA reveals the dietary diversity of the widespread insectivorous predator Daubenton's Bat (Myotis daubentonii) in Southwestern Finland. PLOS ONE. 2013;8:e82168.

59. Rather MA, Willayat MM, Wani SA, Hussain SA, Shah SA. Enterotoxin gene profile and molecular epidemiology of Aeromonas species from fish and diverse water sources. J Appl Microbiol. 2019;127:921-31.

60. Ran C, Qin C, Xie M, Zhang J, Li J, Xie Y, et al. Aeromonas veronii and aerolysin are important for the pathogenesis of motile aeromonad septicemia in cyprinid fish. Environ Microbiol. 2018;20:3442-56.

61. Brito C, Cabanes D, Sarmento Mesquita F, Sousa S. Mechanisms protecting host cells against bacterial pore-forming toxins. Cell Mol Life Sci. 2019;76:1319-39.

62. Wiles TJ, Jemielita M, Baker RP, Schlomann BH, Logan SL, Ganz J, et al. Host gut motility promotes competitive exclusion within a model intestinal microbiota. PLoS Biol. 2016;14:e1002517.

63. Iwai S, Weinmaier T, Schmidt BL, Albertson DG, Poloso NJ, Dabbagh K, et al. Piphillin: improved prediction of metagenomic content by direct inference from human microbiomes. PLOS ONE. 2016;11:e0166104.

64. Sugita H, Miyajima C, Deguchi Y. The vitamin B12-producing ability of the intestinal microflora of freshwater fish. Aquaculture. 1991;92:267-76.

65. Hellgren LI. Occurrence of bioactive sphingolipids in meat and fish products. Eur J Lipid Sci Technol. 2001;103:661-7.

66. Kjær MA, Ruyter B, Berge GM, Sun Y, Østbye T-KK. Regulation of the omega-3 fatty acid biosynthetic pathway in Atlantic salmon hepatocytes. PLoS ONE. 2016;11:e0168230.

67. Costantini L, Molinari R, Farinon B, Merendino N. Impact of omega-3 fatty acids on the gut microbiota. Int J Mol Sci. 2017;18:2645.

68. Alberdi A, Aizpurua O, Gilbert MTP, Bohmann K. Scrutinizing key steps for reliable metabarcoding of environmental samples. Methods Ecol Evol. 2017;00:1-14.

69. Muyzer G, de Waal EC, Uitterlinden AG. Profiling of complex microbial populations by denaturing gradient gel electrophoresis analysis of polymerase chain reaction-amplified genes coding for 165 rRNA. Appl Environ Microbiol. 1993;59:695-700.

70. Caporaso JG, Lauber CL, Walters WA, Berg-Lyons D, Lozupone CA, Turnbaugh PJ, et al. Global patterns of 16 S rRNA diversity at a depth of millions of sequences per sample. Proc Natl Acad Sci U S A. 2011;108(Suppl 1):4516-22

71. Murray DC, Coghlan ML, Bunce M. From benchtop to desktop: important considerations when designing amplicon sequencing workflows. PLOS ONE. 2015;10:e0124671.

72. Bustin SA, Benes V, Garson JA, Hellemans J, Huggett J, Kubista M, et al. The MIQE guidelines: minimum information for publication of quantitative real-time PCR experiments. Clin Chem. 2009;55:611-22.

73. Shapiro B, Hofreiter M, La J, Er A. Ancient DNA: methods and protocols. New York: Humana Press; 2012.

74. Binladen J, Gilbert MTP, Bollback JP, Panitz F, Bendixen C, Nielsen R, et al. The use of coded PCR primers enables high-throughput sequencing of multiple homolog amplification products by 454 parallel sequencing. PLOS ONE. 2007;2:e197.

75. DeAngelis MM, Wang DG, Hawkins TL. Solid-phase reversible immobilization for the isolation of PCR products. Nucleic Acids Res. 1995:23:4742-3.

76. Rohland N, Reich D. Cost-effective, high-throughput DNA sequencing libraries for multiplexed target capture. Genome Res. 2012;22:939-46.

77. Carøe C, Bohmann K. Tagsteady: a metabarcoding library preparation protocol to avoid false assignment of sequences to samples. bioRxiv. 2020. https://doi.org/10.1101/2020.01.22.915009. 
78. Carøe C, Gopalakrishnan S, Vinner L, Mak SST, Sinding MHS, Samaniego JA, et al. Single-tube library preparation for degraded DNA. Methods Ecol Evol. 2018:9:410-9.

79. Schubert M, Lindgreen S, Orlando L. AdapterRemoval v2: rapid adapter trimming, identification, and read merging. BMC Res Notes. 2016;9:88.

80. Martin M. Cutadapt removes adapter sequences from high-throughput sequencing reads. EMBnet.journal. 2011:17:10-2.

81. Callahan BJ, McMurdie PJ, Rosen MJ, Han AW, Johnson AJA, Holmes SP. DADA2: High-resolution sample inference from Illumina amplicon data. Nat Methods. 2016;13:581-3.

82. Dessau RB, Pipper CB. R"-project for statistical computing. Ugeskr Laeger. 2008;170:328-30.

83. Narayan NR, Weinmaier T, Laserna-Mendieta EJ, Claesson MJ, Shanahan F, Dabbagh K, et al. Piphillin predicts metagenomic composition and dynamics from DADA2-corrected 16S rDNA sequences. BMC Genomics. 2020;21:56.

84. Love MI, Huber W, Anders S. Moderated estimation of fold change and dispersion for RNA-seq data with DESeq2. Genome Biol. 2014;15:550.

85. Wickham H. ggplot2: Elegant Graphics for Data Analysis. Berlin: Springer; 2016.

86. Kuhn $M$, et al. Building predictive models in $\mathrm{R}$ using the caret package. J Stat Softw. 2008;28:1-26.

87. Florkowski CM. Sensitivity, specificity, receiver-operating characteristic (ROC) curves and likelihood ratios: communicating the performance of diagnostic tests. Clin Biochem Rev. 2008;29(Suppl 1):S83-7.

88. Alberdi A, Gilbert MTP. A guide to the application of Hill numbers to DNA based diversity analyses. Mol Ecol Resour. 2019;19:804-17.

89. Oksanen J, Blanchet FG, Kindt R, Legendre P, Minchin PR, O'hara $\mathrm{RB}$, et al. Package "vegan." Community ecology package, version. 2013;2:1-295.

90. Leff JW. mctoolsr: microbial community data analysis tools. available at github com/leff/mctoolsr. 2016.
91. Lahti L, Shetty S. "microbiome R package." R package version. 20122019. http:microbiome.github.com microbiome.

92. Shen W, Le S, Li Y, Hu F. SeqKit: A cross-platform and ultrafast toolkit for FASTA/Q file manipulation. PLoS ONE. 2016;11:e0163962.

93. Li H, Durbin R. Fast and accurate short read alignment with BurrowsWheeler transform. Bioinformatics. 2009;25:1754-60.

94. Korem T, Zeevi D, Suez J, Weinberger A, Avnit-Sagi T, Pompan-Lotan M, et al. Growth dynamics of gut microbiota in health and disease inferred from single metagenomic samples. Science. 2015;349:1101-6.

95. Brown CT, Olm MR, Thomas BC, Banfield JF. Measurement of bacterial replication rates in microbial communities. Nat Biotechnol. 2016;34:1256-63.

96. Li D, Liu C-M, Luo R, Sadakane K, Lam T-W. MEGAHIT: an ultra-fast singlenode solution for large and complex metagenomics assembly via succinct de Bruijn graph. Bioinformatics. 2015;31:1674-6.

97. Hyatt D, Chen G-L, Locascio PF, Land ML, Larimer FW, Hauser LJ. Prodigal: prokaryotic gene recognition and translation initiation site identification. BMC Bioinform. 2010;11:119.

98. Kanehisa M, Sato Y, Morishima K. BlastKOALA and GhostKOALA: KEGG tools for functional characterization of genome and metagenome sequences. J Mol Biol. 2016;428:726-31.

99. Davis NM, Proctor DM, Holmes SP, Relman DA, Callahan BJ. Simple statistical identification and removal of contaminant sequences in marker-gene and metagenomics data. Microbiome. 2018;6:226.

100. Benjamini Y, Hochberg Y. Controlling the False Discovery Rate: A Practical and Powerful Approach to Multiple Testing. J R Stat Soc Series B Stat Methodol. 1995;57:289-300.

\section{Publisher's Note}

Springer Nature remains neutral with regard to jurisdictional claims in published maps and institutional affiliations.
Ready to submit your research? Choose BMC and benefit from:

- fast, convenient online submission

- thorough peer review by experienced researchers in your field

- rapid publication on acceptance

- support for research data, including large and complex data types

- gold Open Access which fosters wider collaboration and increased citations

- maximum visibility for your research: over 100M website views per year

At BMC, research is always in progress.

Learn more biomedcentral.com/submissions 